\title{
ENSINO DE GEOMETRIA ANALÍTICA: ALGUNS PRESSUPOSTOS DA SEQUÊNCIA FEDATHI NO CONTEXTO DA FORMAÇÃO DE PROFESSOR DE MATEMÁTICA
}

\author{
Francisco Regis Vieira Alves \\ Instituto Federal do Ceará, câmpus Fortaleza \\ Fortaleza, Ceará \\ E-mail: fregis@ifce.edu.br
}

\section{Ana Carolina Costa Pereira \\ Universidade Estadual do Ceará \\ Fortaleza, Ceará \\ E-mail: carolina.pereira@uece.br}

Resumo: Nesse artigo são discutidas situações didáticas envolvendo a aplicação dos pressupostos que caracterizam as fases de ensino da Sequência Fedathi - SF. Seu contexto de aplicação envolve a Geometria Analítica. Desse modo, na seção inicial, indicam-se certos entraves pertinentes à qualidade do livro didático. Em seguida, descrevem-se as fases de ensino da SF, acentuando caracteres específicos requeridos no ensino desse conteúdo. Por fim, são trazidas três situaçõesproblema conhecidas evidenciando vários elementos que emergem da relação professor-alunosaber, com o auxílio do software GeoGebra. Na ausência de uma metodologia de base, no entanto, algumas das relações discutidas podem passar despercebidos ao professor de Matemática pouco experiente.

Palavras-chave: ensino. geometria analítica. sequência Fedathi. professor de matemática.

\section{ANALITHICAL GEOMETRY TEACHING: AN DISCUSSION ABOUT THE FEDATHI SEQUENCE'S ASSUMPTIONS IN THE MATHEMATICAL TEACHING INSTRUCTION}

Abstract: This article discusses the teaching of situations involving the application of the assuptions that characterize the educational stages of Sequence Fedathi - SF. Your application context involves analytic geometry, thus, in the initial section; we indicated certain obstacles pertaining to the quality of textbook. We bring three known problem situations and we demonstrated various elements that emerge from the relationship teacher - pupil - knowledge, with the GeoGebra's help. However, in the absence of a baseline methodology some of these relationships, may go unnoticed in the eyes of the inexperienced teacher of mathematics.

Keywords: teaching. analythical geometry. fedathi sequence. mathematics teacher.

Recebido em 30/03/2015. Publicado em 30/06/2016. 


\section{INTRODUÇÃO}

Nesse trabalho, discutimos algumas aplicações da metodologia de ensino nominada Sequência Fedathi - SF. Ademais, além de descrever algumas de suas aplicações e implicações para o ensino e a mediação do futuro professor de Matemática, bem como o professor iniciante ${ }^{1}$ no ofício, destacamos também elementos preocupantes vinculados ao ensino de Geometria Analítica, sobretudo relacionados ao livro didático, como o elemento constituinte principal de sua praxis em sala de aula.

O livro didático, que constitui um dos principais instrumentos para o exercício do ofício do professor de Matemática em sala de aula, pode condicionar/afetar hábitos inadequados aos aprendizes. Desse modo, uma razoável visão de mediação, não apenas de conteúdo, mas também de transmissão e transposição de um saber específico em sala de aula, pode sugerir caminhos para a superação de determinados entraves. Vejamos inicialmente, porém, obstáculos intrínsecos (de natureza epistemológica) ao conteúdo de Geometria Analítica.

\section{O ENSINO DE GEOMETRIA ANALÍTICA}

Quando buscamos indicar os elementos que evidenciamos serem preocupantes, relacionados ao ensino de Geometria Analítica (ANDRADE, 2007; SANTOS, 2011; GUEDES, 2013; SANTOS, 2008; SOARES, 2013), possivelmente, um particular que se destaca se refere ao livro didático. De fato, esse instrumento desempenha o papel principal quando nos atemos aos suportes que apoiam a mediação do professor em sala de aula. O que sabemos, todavia, sobre a sua qualidade? Sendo ele um dos principais instrumentos didáticos do professor, até que ponto sua qualidade pode comprometer a transmissão de saberes pelo docente? Perguntas como essas são pertinentes, na medida em que deparamos as análises de Lima e outros (2001, p. 210) que indicam

[...] a experiência didática tem mostrado que a condição de alinhamento de três pontos, dada em forma de um determinante, apesar de ser uma bonita fórmula, não deve ter o destaque que tem no livro (e em muitos outros), pois desse modo

\footnotetext{
${ }^{1}$ Nesse trabalho, quando nos referimos ao professor iniciante, empregamos as concepções de Ponte \& Oliveira (2002, p. 151-152) que descrevem o conhecimento profissional como um conhecimento orientado para as situações de prática. Assim, um professor iniciante se caracteriza como aquele que possui um limitado conhecimento didático, o qual constitui uma das vertentes do conhecimento profissional.
} 
Alves e Pereira, 2016 - Formação Inicial e Continuada

acaba funcionando como a receita de bolo favorita em matéria de equação de reta, escondendo os significados dos termos envolvidos.

Um pouco mais adiante, os autores acentuam, ainda que:

[...] a apresentação das equações paramétricas de uma reta é feita sem nenhuma motivação e sem nenhuma aplicação interessante em que o parâmetro pudesse ter uma interpretação geométrica ou física (por exemplo, o tempo). O leitor fica sem saber porque inventaram equações paramétricas e para que elas servem. Mais uma vez a abordagem vetorial também faz falta (LIMA e outros, 2001, p. 211).

Essas considerações fornecem alguns indícios que respondem, pelo menos em parte, nossas duas questões iniciais. Destarte, além de utilizar um instrumento didático de qualidade duvidosa, as consequências em sua metodologia e a ausência de motivação no leitor (e nos alunos) podem ser preocupantes. Seguiremos, pois, as orientações de Lima e outros (2001) e Lima (1999; 2005). Assim, exploraremos, na medida do necessário, a noção de vetores.

Em grande parte, o livro didático é o responsável pelo discurso oficial adotado em sala de aula. Assim, o professor de Matemática deve ficar atento ao uso da Língua Portuguesa no contexto do ensino de Geometria Analítica. Por exemplo, quando afirmamos que "todo plano separa o espaço", do ponto de vista matemático, o verbo "separar" quer significar, que dado um plano $\Pi$, se retiramos o plano, esse espaço $E$ fica decomposto em dois semi-espaços determinados por esse plano. Escrevemos $E-\Pi=S \bigcup_{\substack{\text { união } \\ \text { disiunta }}} S^{\prime}$. Outra expressão do tipo "pé da reta" para descrever a simbologia $r \perp \Pi$, formalmente, significa que uma reta $r$ é perpendicular a qualquer reta contida nesse plano.

Dizemos, sem muito cuidado, em sala de aula que "um ponto $\mathrm{P}$ está sobre uma reta $r$ ", mas, de fato, significa dizer que o ponto $\mathrm{P}$ é um elemento do conjunto $r$. Outra expressão que requer atenção do professor iniciante diz respeito ao sentido da frase "A reta $r$ pertence ao plano $\Pi$ ". Matematicamente, isto não é verdade, pois uma reta $r$ é um conjunto de pontos, o que pertence ou não ao plano $\Pi$ são os pontos. Desse modo, o correto afirmar é que "uma reta $r$ esta contida num plano $\Pi$ ". Outro exemplo que não se pode confundir é descrito por afirmações do tipo: "a 
reta $r$ intercepta o plano $\Pi$ ", quando o certo é mencionar que "a reta $r$ intersecta o plano $\Pi$ ". A inconsistência aqui - apesar de que, num sentido metafórico, descrevemos o mesmo episódio envolvendo uma reta e um plano - porém, do mau emprego do verbo "interceptar" que difere, nesse caso, do verbo "intersectar".

Um dos elementos delicados no ensino de Matemática diz respeito à manifestação de definições formais $^{2}$ que implicam "raciocínios circulares ou elípticos". Possivelmente, o caso mais clássico concerne à definição de número irracional. No contexto do ensino, não é pouco comum depararmos explicações segundo as quais "um número irracional é um número que não é racional". Consequentemente, um número racional é "um número que não é irracional".

Além de equivocado e limitado, tal argumentação pode surgir em razão da ingênua propriedade $I R=\square \bigcup(I R-\square)$ discutida no contexto escolar. No âmbito de ensino da Geometria Analítica, não nos livramos de concepções desta natureza. Por exemplo, o que obteremos como resposta dos aprendizes quando requisitamos que forneçam o significado de retas ortogonais? Na maioria dos casos, ouvimos explicações dando conta de que "são retas que formam entre si um ângulo reto.", quando, porém, perguntamos o que é um ângulo reto, ouvimos, frequentemente: “Ah professor! É o ângulo formado entre duas retas ortogonais".

Uma noção de entendimento difícil, desde o tempo dos helênicos, refere-se à ideia de existência. Quando falamos de existência, podemos declarar: (a) há um processo matemático; (b) existe um objeto matemático. No primeiro caso, de modo tradicional, declarar a existência de um processo matemático condiciona a noção de compreendermos que determinado processo matemático é exequível, que não apresenta contradições e que garante alcançarmos certo resultado. Por exemplo, podemos definir a soma de dois vetores ${ }^{3} v$ e w por meio de dois processos.

Na primeira definição, o processo matemático que permite descrever $v+w$ consiste em representar $v=\overrightarrow{A A^{\prime}}$ e, em seguida, representar $w=\overrightarrow{A^{\prime} A^{\prime \prime}}$ por um segundo segmento orientado

\footnotetext{
${ }^{2}$ Soares (2013, p. 108) assinala a preocupação com definições nos conteúdos de apostilas de Geometria Analítica, entre as décadas de 60 e 70 . Outro fator evidenciado pelo autor diz respeito a presença constante de figuras como forma de ilustrar as explicações.

${ }^{3} \mathrm{O}$ estudo de vetores faz parte do programa do Estado do Rio de Janeiro. Apesar de um apelo intuitivo, quando atribuímos às características módulo, direção e sentido, quando falamos de um vetor, consideramos uma infinidade de segmentos com as mesmas características. Assim, a esse conjunto ou classe com esses segmentos, empregamos a notação $\overrightarrow{A B}$.
} 
cujo início seja a extremidade final $A^{\prime}$ do primeiro segmento e por $v+w=\overrightarrow{A A^{\prime \prime}}$ (LIMA e outros, 1999, p. 59). Temos a segunda possibilidade de descrever o processo que caracteriza a mesma definição de soma $v+w$, quando representamos os vetores $v=\overrightarrow{A A^{\prime}}$ e $w=\overrightarrow{A C}$ por segmentos orientados com o mesmo início e definir $v+w=w=\overrightarrow{A D}$, onde $A D$ é a diagonal do paralelogramo que tem dois lados consecutivos iguais a $A A^{\prime}$ e $A C$ (LIMA e outros, 1999, p. 59). Reparemos que a primeira definição de soma proporciona maior generalidade da noção, ao passo que, na segunda, não podemos prescindir de um paralelogramo (os segmentos $A A^{\prime}$ e $\mathrm{AC}$ formam um ângulo nãonulo). No caso (b), quando declaramos que existe um objeto matemático, nos vemos na obrigação de investigar a unicidade desse objeto conceitual. Em outros casos, não contamos com a unicidade referente a determinadas propriedades que caracterizam um objeto ou uma noção. Por exemplo, quando tomamos duas retas paralelas $r \mathrm{es}$, podemos escolher qualquer segmento $P Q$ (onde $P \in r \mathrm{e} Q \in \mathrm{s})$, entre as retas paralelas, de modo que temos a distância $d(Q, r)=d(P, s)$ (designam a distância de uma reta a um ponto).

Se, todavia, temos duas retas $r$ e s reversas, podemos obter um só segmento que realiza a menor distância entre essas retas, pela aplicação do teorema de Pitágoras. Outra noção que fornece complicações diz respeito à correspondência biunívoca que pode ser estabelecida entre qualquer eixo $\mathrm{E}$ com o conjunto $I R$ dos números reais, ao fazermos corresponder a origem $\mathrm{O}$ com $\mathrm{O}$ número zero. Em seguida, consideramos que a cada ponto $X \in E$ situado à direita de $\mathrm{O}$ corresponde o número real positivo $x=d(O, X)$. Aos pontos situados à esquerda de 0 correspondem aos números reais negativos, e os designamos por $x=-d(O, X)$. Com um argumento semelhante, estabelecemos uma correspondência biunívoca $\Pi \rightarrow I R^{2}$, onde a cada ponto $P$ de um sistema de coordenadas escolhido arbitrariamente $\Pi$ corresponde um par ordenado $(x, y) \in I R^{2}$. A menção que fizemos às duas correspondências biunívocas que nos permitem tratar de modo indistinto números reais e pontos no eixo orientado $E$, ou pontos no plano $P \in \Pi$ com pares ordenados servem, pois, para dois propósitos, como apreciamos no próximo excerto:

O primeiro é o de atribuir um significado geométrico (e com isto da um maior conteúdo intuitivo) a fatos de natureza numérica, como o comportamento de uma função real de uma variável real, que ganha muito em clareza quando se olha 
Alves e Pereira, 2016 - Formação Inicial e Continuada

para seu gráfico. O segundo propósito do uso das coordenadas vai no sentido oposto recorre-se a elas afim de resolver problemas de Geometria. Esse é o objetivo da Geometria Analítica. No primeiro caso, a ênfase recai sobre a correspondência $I R^{2} \rightarrow \Pi$ e no segundo caso sobre sua inversa $\Pi \rightarrow I R^{2}$. Na prática, esses dois pontos de vista se entrelaçam: para estabelecer os fatos iniciais da Geometria Analítica usam-se os resultados básicos da Geometria Euclidiana (LIMA e outros, 1999).

O que merece ser destacado no longo excerto extraído de Lima e outros $(1999$, p. 6) refere-se às implicações que devemos retirar para a formação do professor de Matemática. De modo particular, observamos que é difícil o docente adquirir um "ponto de vista" flexível, local e, ao mesmo tempo global da própria disciplina, sem contar com um bom domínio sólido de conteúdo, todavia, para um exercício efetivo do ofício de professor, resta a evolução de um cuidado referente a uma transmissão (transposição) eficiente do conhecimento matemático. Na próxima seção, vamos nos deter em preocupações desta natureza e, acentuar uma visão de mediação/transmissão de ensino que não permite negligenciar elementos de ordem epistemológica e outros de natureza diferenciada dos que apresentamos nesta parte inicial.

\section{METODOLOGIA DE ENSINO}

Registramos alguns trabalhos (ALVES \& BORGES NETO, 2009; ALVES; BARRETO \& BORGES NETO, 2011; ALVES \& BORGES NETO, 2012) que envolvem a aplicação da Sequência Fedathi - SF, tanto no contexto escolar como no locus acadêmico. Nesses escritos, notamos preocupações específicas acerca do ensino e da aprendizagem de conceitos matemáticos. Nesta seção, discutiremos a aplicação da SF, como perspectiva de abordagem metodológica aos conceitos de Geometria Analítica.

Assim, com amparo nas preocupações e nos elementos apontados na seção anterior, descrevemos as seguintes fases ou momentos da mediação envolvendo a tríade aluno-professor-saber . Possivelmente, o momento no qual o professor iniciante pode enfrentar maiores dificuldades refere-se ao início de sua aula. Aconselha a boa didática que uma aula de Matemática com qualidade tem início na enunciação de um problema interessante, todavia, como apontamos nas seções anteriores, nem sempre o livro didático detém uma qualidade que possa suprir todas as 
necessidades do professor e assumir concretamente, um estilo de abordagem heurística (POLYA, 1967) dos conteúdos.

Outro embaraço não menos importante é indicado por Sauriau (1881, p. 17) quando acentua: [...] sabemos que condições devem submeter a ideia desejada ou procurada, entretanto, não sabemos quais ideias nos conduzirão a mesma. Assim, malgrado ter um bom domínio do conteúdo específicos e conhecer os argumentos lógicos finais que determinam a verdade ou a falsidade do encontrado, o professor de Matemática deve ficar atento com respeito ao surgimento de ideias tácitas, intuitivas e inesperadas por parte dos seus aprendizes, tendo em vista o engajamento numa situação problema. Assim, indicamos a primeira fase.

\subsection{Fase 1, Tomada de posição: apresentação do problema}

No âmbito da Geometria Analítica, o professor de Matemática não deve transmitir aos seus estudantes crenças equivocadas, como, por exemplo, a ideia de que a Geometria Analítica serve apenas para resolver problemas de Geometria Analítica. Desse modo, dos primeiros aspectos a serem observados são a ideia e a estruturação adequada de enunciados que não devem, necessariamente, informar aos aprendizes que o problema se refere à Geometria Analítica. Nesta fase de ensino, é imprescindível a elaboração de desenhos adequados, de modo que os dados pertinentes aos enunciados possam ser identificados nas figuras ou desenhos produzidos pelos estudantes. A introdução precipitada de um sistema notacional também não é indicada.

São recomendáveis, por exemplo, a comparação dos desenhos produzidos pelos aprendizes e a eleição do desenho que melhor traduz a situação prevista no enunciado. Além disso, as imagens que concebemos a cada momento não surgem do caos e sim de um pensamento anterior (SAURIAU, 1881, p. 12). Desse modo, toda a produção dos desenhos deve ser reaproveitada na próxima fase de ensino e, nela, a partir da evolução dos aprendentes, um sistema notacional que proporcionará a homogeneização das informações trocadas entre o grupo é aconselhável.

\subsection{Fase 2, Maturação: entendimento e identificação das variáveis envolvidas no problema}

No ensino de Geometria Analítica, na medida em que evitamos algebrizar a investigação, os alunos devem identificar as variáveis mais importantes com suporte na comparação entre os 
dados no enunciado e a figura produzida da etapa anterior. De outra parte, a tecnologia pode indicar elementos inexequíveis de exploração na etapa anterior. Assim, a emprego de algum software (como o Geogebra) pode oferecer um bom entendimento e a identificação da melhor estratégia de solução.

Vale destacar, ainda, que a exploração adequada do software pode inserir, no contexto do debate em sala de aula, a noção do erro e a produção de conjecturas. É importante, com efeito, ficarmos atentos ao fato de que devem desaparecer as contradições sobre as quais pode declinar inevitavelmente nosso raciocínio. Uma mediação adequada do erro, entretanto, como consequência de contradições ou inconsistência no raciocínio, pode adquirir um valor didático importante. Assim, sugerimos o uso, no caso da Geometria Analítica, de construções geométricas que se assemelham, mas não exatamente as mesmas previstas nos teoremas. Com isto, podemos proporcionar um clima propício ao surgimento da incerteza e do erro, em contraposição ao sentimento de certeza matemática.

\subsection{Fase 3, Solução: apresentação e organização de esquemas/modelos que visem à solução do problema}

Muitos problemas de Geometria Analítica expressam uma correlação muito próxima ao campo da Geometria Plana. Assim, apesar de a estratégia testada nesta fase se fundamentar na Geometria Analítica, na próxima fase, o professor pode comparar, relacionar e conduzir seus aprendizes a observar as relações conceituais entre Geometria Analítica e Geometria Plana.

Sublinhamos que nesta fase o aluno deve empregar um raciocínio, semelhante ao descrito por Gibel \& Brousseau (2005, p. 17), do tipo $p \stackrel{\text { Regra }}{\rightarrow} q$, onde $p$ eq constituem propriedades ou declarações caracterizadas por sentenças proposicionais e uma "regra" que possibilita declarar esta inferência como verdadeira ou falsa. Apesar de que a palavra "raciocínio" não se refere a um domínio estrito da Matemática ou da Lógica Clássica (GIBEL \& BROUSSEAU, 2005, p. 15), algumas formas de raciocínio podem ser descritos em termos de uma ou da outra. Por exemplo, na fase de solução, o aluno pode empregar um raciocínio por redução ao absurdo que, em termos do cálculo

proposicional equivale a se obter $H \rightarrow T \Leftrightarrow(H \wedge \sim T) \rightarrow f$. Nesse caso, tomamos $H$ : hipótese e T:tese . Observamos que esse modelo lógico pode ser empregado para a solução de 
uma situação problema, todavia, o aluno não ser consciente do porque de sua aplicabilidade e dos limites de consistência.

\subsection{Fase 4, Prova ${ }^{4}$ : formalização do modelo matemático a ser ensinado}

Problemas interessantes de Geometria Analítica possuem, de modo subjacente, modelos formais que permitem a generalização dos argumentos e atribuem o caráter de veracidade das soluções obtidas. Sublinhamos o fato de que o professor deve conhecer demonstrações analíticas e sintéticas que conduzem aos mesmos resultados.

Assim, poderá desenvolver um entendimento conceitual dos aprendizes, na medida em que estimula as relações entre Geometria Plana e Geometria Analítica. Por fim, o professor deve ficar atento, uma vez que, se temos um problema geométrico (que não menciona coordenadas) e queremos resolvê-lo usando Geometria Analítica, temos a liberdade de introduzir no plano o sistema de coordenadas que acharmos mais conveniente para nosso problema (LIMA e outros, 1999, p.19).

Devemos reconhecer que, na perspectiva apontada por Lima e outros (1999, p. 19), não se constitui como uma habilidade automática e flexível, quando falamos do professor de Matemática iniciante. O docente experimentado, porém, deve possuir no stock de dados, situações diferenciadas e variadas, nas quais, ele consegue antever as possibilidades indicadas por Lima e outros (1999) e Lima (2005, p. 31).

Ainda no momento de prova, vale colocar em evidência a expressão cunhada por Souriau (1881) como de "pensar de lado" ou "pensamento lateral", ocorrente quando, ao decorrer de uma dedução, tendo em vista a maior importância do caráter operatório das inferências lógicas (Se... então...) utilizadas em uma demonstração em detrimento do seu conteúdo, o professor pode desenvolver esses passos de inferência e, concomitantemente, com arrimo do raciocínio intuitivo, antever um possível erro ou resultado indesejado rumo à tese.

\footnotetext{
${ }^{4}$ Balacheff (1988, p. 29-30) diferencia uma prova de uma demonstração. A primeira noção se caracteriza na medida em que uma explicação é reconhecida e aceita por consenso. A passagem de uma explicação a uma prova se da por uma referência social e local. Por outro lado, a demonstração caracteriza um gênero de discurso na forma estritamente codificada e algumas de suas passagens podem ser omitidas e deixadas a cargo do leitor. Na SF o professor deve priorizar situações didáticas que envolvam a primeira noção.
} 
Alves e Pereira, 2016 - Formação Inicial e Continuada

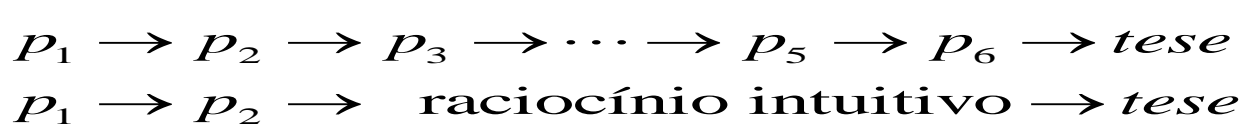

Por fim, concordamos com Sauriau (1881, p. 38), quando menciona que a lógica não cria a verdade. Assim, a preocupação do professor de Matemática não pode se restringir ao domínio de aplicação de uma demonstração, afinal, o estímulo ao raciocínio não se restringe à apresentação de modelos matemáticos formais (BROUSSEAU \& GIBEL, 2005, p. 14). Sua ação é desenvolvida a fim de obter o convencimento e engajamento dos iniciantes. Nesta perspectiva, embora possamos empregar todos os artifícios lógicos para se atingir o resultado almejado da Geometria Analítica, o docente precisa convencer seus alunos sobre a confiabilidade dos dados.

A verdade matemática, com efeito, uma vez alcançada em sala de aula, não deve ser encarada como uma questão de lógica e, sim como um ponto de consenso e resultado do debate em sala de aula. Na próxima seção, trazemos três problemas de aplicação e sugestão de uso em sala de aula. Vale sublinhar que não buscamos o caráter de ineditismo das situações, mas o alcance de uma visão de complementaridade, com base na ótica de um olhar fundamentada na SF e o uso da tecnologia, consubstanciada pelo uso do software GeoGebra.

\section{APLICAÇÕES}

Nesse segmento do ensaio, vamos discutir de modo específico três situações-problema abordadas nas vídeoaulas (WAGNER, 2006; 2011) referentes ao Programa de Aperfeiçoamento para Professores de Matemática do Ensino Médio - PAPEM. Colocamos em evidência parte do conteúdo de Geometria Analítica dessas videoaulas ${ }^{5}$ (2008/2010) tendo em conta as preocupações expressas na sequência: (i) a qualidade e notoriedade dos professores que conduzem as aulas de Geometria Analítica é indiscutível; (ii) a importância das indicações didáticometodológicas registradas ao decorrer de suas mediações; (iii) a inexistência de uma proposta metodológica explícita e definida, adotada nessas aulas; (iv) e inexistência de uma perspectiva sistemática de replicação das estratégias discutidas em outras situações reais de ensino.

Os dois últimos itens requerem alguns comentários adicionais. Inicialmente, enquanto identificamos nessas aulas constantes reflexões e dicas concernentes ao ensino de Geometria

${ }^{5}$ Essas aulas podem ser encontradas no site: http://video.impa.br/index.php?page=download 
Analítica, advogamos a ideia de que essas dicas possuem valor pedagógico indiscutível. Quando, porém, nos reportamos ao professor iniciante, é essencial que esse se familiarize com pressupostos gerais e que devem nortear a aquisição de experiências. Ademais, a exposição magistral de uma pessoa experiente é sempre esperada, entretanto, não se pode garantir que sempre ocorre algum tipo de aprendizagem ou que o entendimento dos alunos corresponda ao desejado. Apresentamos então o primeiro problema discutido em Wagner (2006).

Problema 1. Mostre que as alturas de um triângulo se cortam em um ponto.

Fonte: Wagner (2006).

Esta questão exibe um diferencial, na medida em que permite ao professor de Matemática definir uma noção em sala de aula, sem se apoiar nas práticas ordinárias exploradas pelos livros didáticos, que se precipitam em fornecer as condições lógicas às quais os objetos matemáticos devem satisfazer, antes de explorar o sentido e o significado de uma nova entidade conceitual. Assim, na fase de tomada de posição, não prevemos o fornecimento de uma definição formal.

Em outras palavras, isto informa o professor de Matemática que não se deve começar uma aula por uma definição formal, pois, nesse caso, descrevemos sob o prisma da lógica proposicional, um objeto desprovido de um significado ou sentido, sob o ponto de vista do aluno. Nesta fase, a heurística do raciocínio a ser empregado diz respeito a um modo standard na atividade matemática. De maneira sistemática, a forma de raciocinar aqui é caracterizada pelo fato de se admitir a existência de dois pontos, $H_{1}$ e $\mathrm{H}_{2}$ (redução ao absurdo), distintos e, ao final, se deve concluir que na verdade são iguais.

Nesta fase, o professor pode empregar o pensamento lateral, na medida em que, ante a pluralidade de situações sugeridas pelos alunos, pode propor e não eleger peremptoriamente, o desenho que proporciona melhores condições de se verificar $H_{1}=\mathrm{H}_{2}$. Desse modo, o pensamento intuitivo do docente progride e, em alguns segundos, pode aventar alternativas alvissareiras aos seus alunos. 
Figura 1. Possibilidades de desenhos que devem guiar o raciocínio heurístico na fase de tomada de posição da SF.

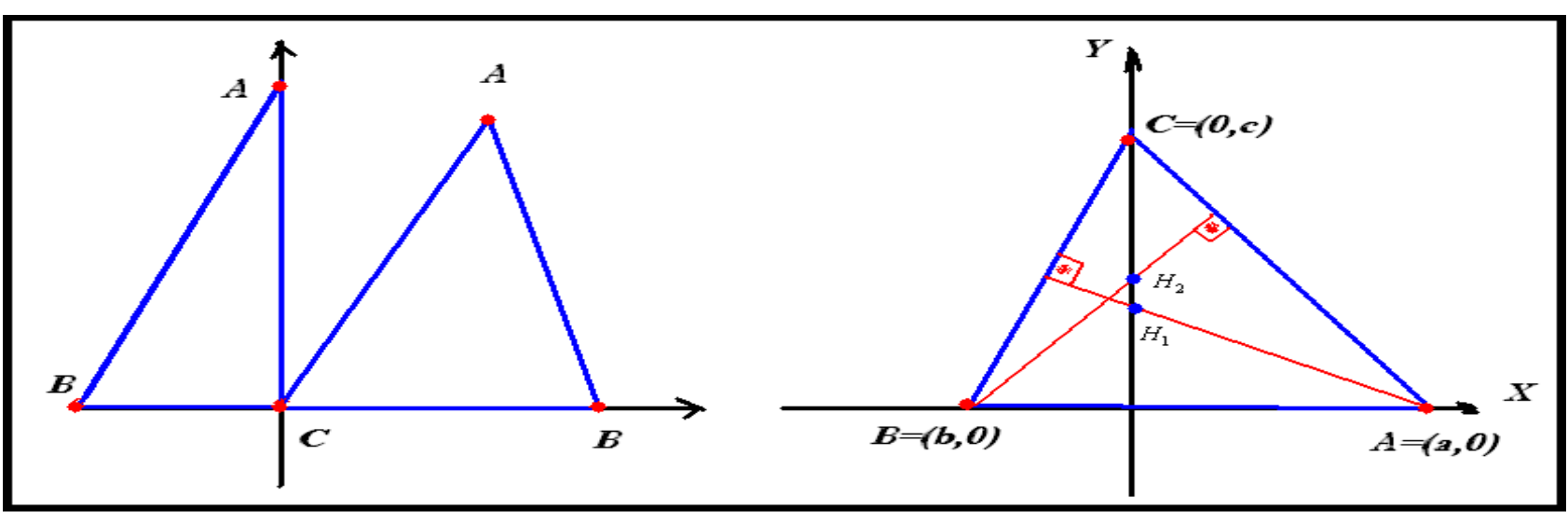

Fonte: Dados da investigação.

Na fase de maturação, por intermédio do debate em sala de aula, um desenho que melhor proporciona a obtenção e aplicação das estratégias, deve ser destacado. De certo, um professor experiente, deverá saber antever os desenhos que podem dificultar a busca de soluções. A percepção há de guiar tal atividade de escolha da figura adequada. Na fase de solução, podemos calcular o coeficiente angular do segmento $B C$, descrito por $m_{B C}=\frac{0-c}{b-0} \therefore m_{r}=\frac{b}{c}$. ̇̀ possível descrever agora a reta $\mathrm{r}$ por $y-0=\frac{b}{c}(x-a) \rightarrow y=\frac{b}{c} x-\frac{a b}{c}$. Reparemos, porém, que a reta $\mathrm{r}$ intersecta o eixo OY no ponto $H_{1}=(0, h) \in r \therefore h=-\frac{a b}{c}$. Agora, para o coeficiente $m_{A C}=\frac{0-c}{a-0}=-\frac{c}{a}$, portanto, a reta s é descrita por $s: y-0=\frac{a}{c}(x-b)$. Vemos então que a intersecção $H_{2}=\left(0, h^{\prime}\right)$, assim, teremos $h^{\prime}-0=\frac{a}{c}(0-b) \leftrightarrow h^{\prime}=-\frac{a b}{c} ;$ por fim, $H_{1}=(0, h)=H_{2}$.

Mas assim contrariamos o fato que assumimos na fase de tomada de posição. Sinteticamente, o professor pode apresentar aos estudantes o seguinte desenho. Na figura 2, consideramos duas alturas relativas aos lados do $\triangle A C Q$; como vimos no problema anterior, seu ponto único de intersecção será o ortocentro. 
Figura 2. Na fase de prova o professor pode comparar os modelos formais da Geometria Analítica com a Geometria Plana.
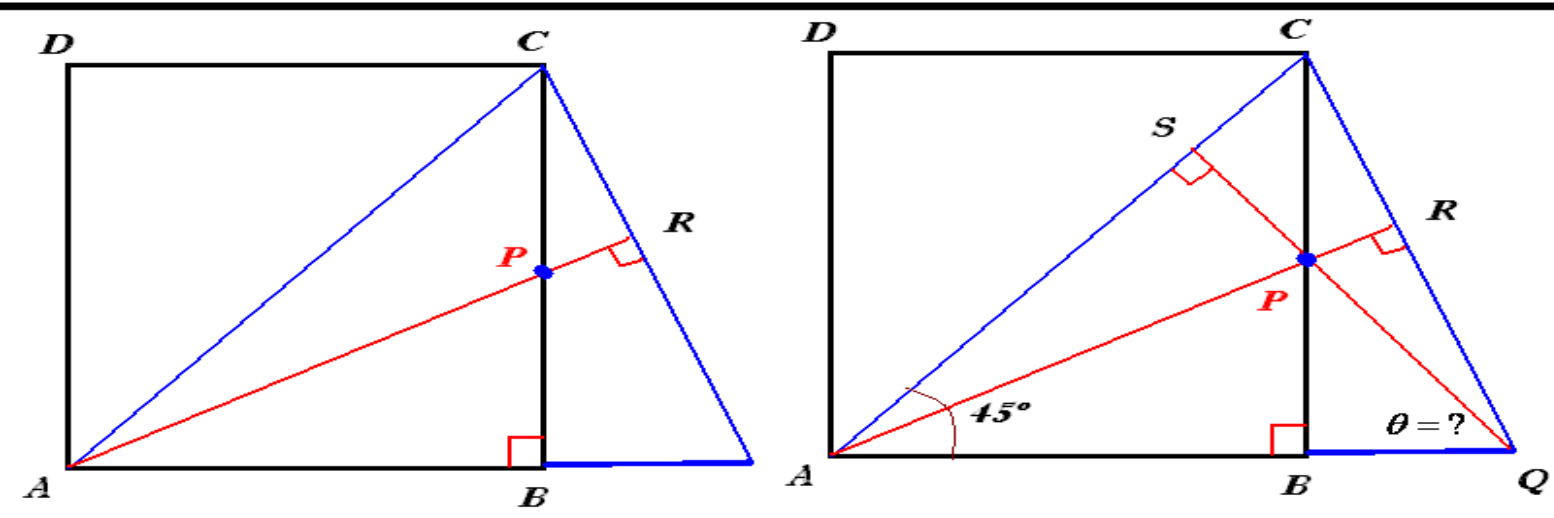

Fonte: Elaboração dos autores

Em seguida, entretanto, quando tomamos a diagonal do quadrado $A B C D$, teremos um ângulo de 45․ Por fim, porém, considerando o $\triangle A S Q$, que possui um ângulo de $45^{\circ}$ e um de 90 , consequentemente, devemos ter $\theta=45^{\circ}$. Na fase de prova, recordamos as considerações de Polya (1973, p, 86), ao explicar que uma definição matemática cria um significado matemático. Assim, é chegado o momento didático ideal para se designar um significado matemático para a palavra "ortocentro".

Notemos que as palavras estão intimamente associadas às ideias (SAURIAU, 1881, p. 121), de tal modo que, ao designar o ortocentro como ponto em comum das alturas relativas a cada um dos lados de um triângulo $\triangle A B C$, ao pronunciarmos esta palavra mais uma vez, propiciamos a oportunidade de imaginar imediatamente uma figura geométrica que associamos a mesma, consequentemente, seu significado é apreendido. Vejamos o segundo problema que envolve uma construção geométrica interessante discutida em Wagner (2009).

Problema 2. Considerando um quadrado ABCD e um ponto $\mathrm{P}$ sobre o segmento de reta $\mathrm{BC}$. Admitindo que a reta que contém $C$ é perpendicular a $A P$, corta $A B$ no ponto $Q$. Prove que o ângulo $B Q P$ é constante.

Fonte: Wagner (2011).

Um dos primeiros obstáculos cognitivos identificados no problema 3 diz respeito ao recurso de um desenho que auxilia/guia a intuição. Notemos em seu enunciado, descrito apenas em termos da 
Alves e Pereira, 2016 - Formação Inicial e Continuada

língua materna, que nada se fala sobre eixos ou pares ordenados. Assim, na tomada de posição, o professor pode instigar seus alunos na investigação e descrição de uma figura adequada, que possibilita a percepção/identificação das variáveis mais pertinentes ao problema.

Na fase de maturação, na dependência do fato de que, por intermédio da atividade dos estudantes, se tenha produzido uma figura conveniente e que proporciona a percepção dos elementos mais importantes nesta situação, o professor pode estimular a produção de conjecturas na forma de sentenças proposicionais à respeito dos elementos que são "dados a ver" na figura produzida.

Nessa fase ainda, os aprendizes são estimulados a compreender os elementos invariantes na construção geométrica. A partir desta discussão, de modo individual ou em grupo, podemos escolher os seguintes pontos: $A=(-1,0), \mathrm{P}=(0, \mathrm{a}), \mathrm{C}=(0,1), \mathrm{Q}=(\mathrm{b}, 0)$. Na figura abaixo sublinhamos uma descrição razoável da situação proposta nesse problema. Reparemos que a mudança do desenho pode condicionar o aumento ou diminuição dos cálculos a serem efetuados nesse caso.

Figura 3. Na fase de maturação do professor pode gerar situações conflitivas que induzem ao erro.

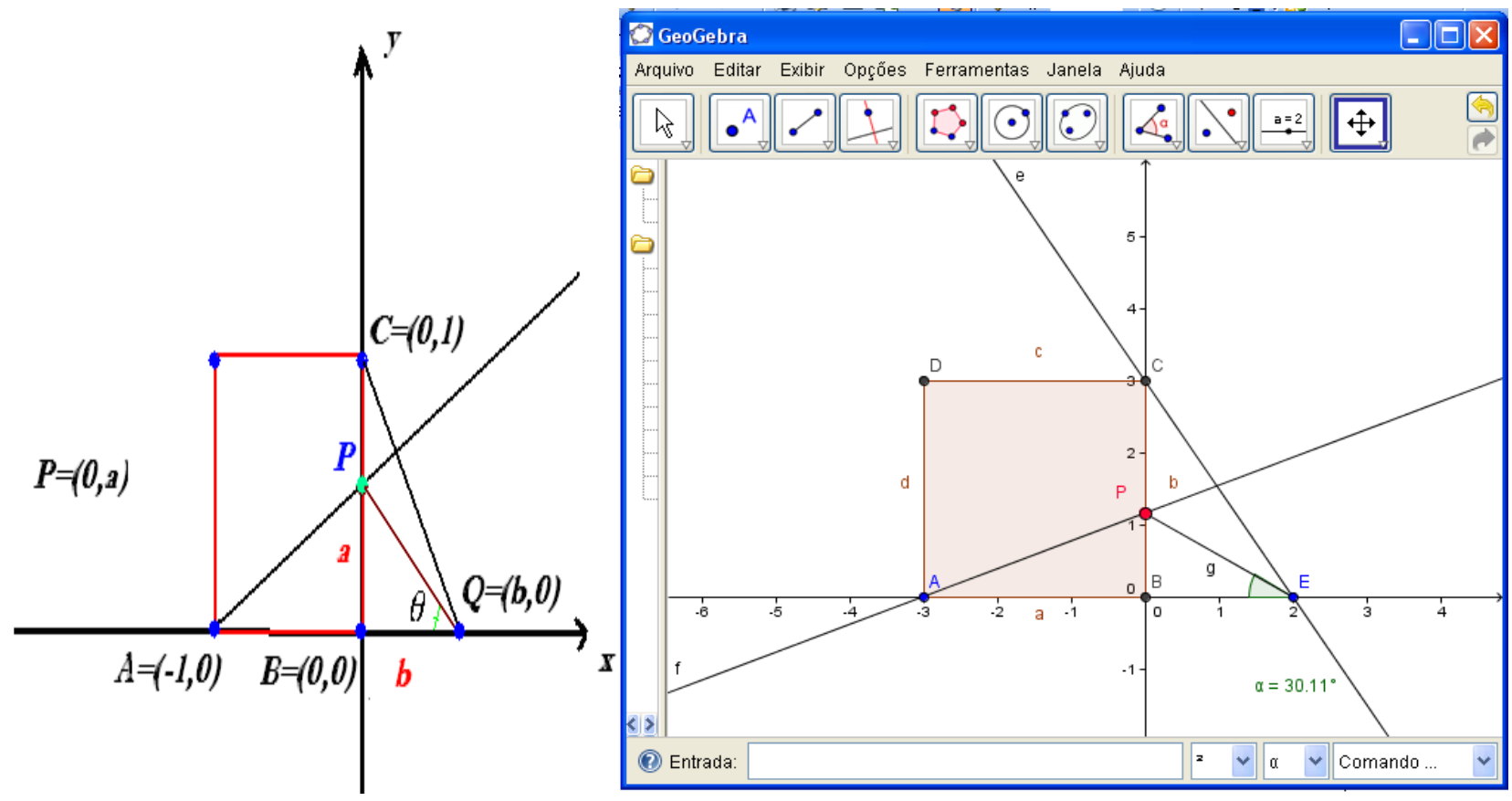

Fonte: Dados da investigação.

Na fase de solução, o professor pode explicitar ou não a possibilidade de solução do problema 3, de modo analítico ou de forma sintética. Assim, o docente pode sugerir a identificação dos vetores $\overrightarrow{A P}$ e $\overrightarrow{\mathrm{CQ}}$ (fig. 3) e concluir que: $\overrightarrow{A P}=(1$, a) e $\overrightarrow{\mathrm{CQ}}=(b,-1)$. Em seguida, empregamos a hipótese da 
condição de perpendicularismo, ou seja, $\overrightarrow{A P} \perp \overrightarrow{\mathrm{CQ}} \leftrightarrow(1, \mathrm{a}) \cdot(b,-1)=0 \leftrightarrow 1 \cdot b+a \cdot(-1)=0 \therefore a=b$

. Na fase de prova, o educador deve destacar o dado que pode ter sido obtido, entretanto, não compreendido pelos educandos. Verifiquemos que, para todo ponto $P \in B C$, com uma reta que contém $\mathrm{C}$ e é perpendicular ao segmento $\mathrm{AP}$, determina os pontos, e os vetores $\overrightarrow{A P}=(1, a)$ e $\overrightarrow{\mathrm{CQ}}=(b,-1)$, com $a=b$. Isto nos diz, contudo, que o triângulo $\triangle B P Q$ é sempre isósceles, independentemente da posição do ponto P. Na figura 4, destacamos uma construção básica envolvida nesse problema realizada com o Geogebra. O caráter de invariância do ângulo $B Q P$ pode ser compreendido de um modo completamente diferenciado, em relação ao ensino restrito às mídias lápis e papel.

Figura 4. Na fase de prova o resultado obtido na fase de solução pode ser confrontado com dados produzidos pelo modelo computacional.

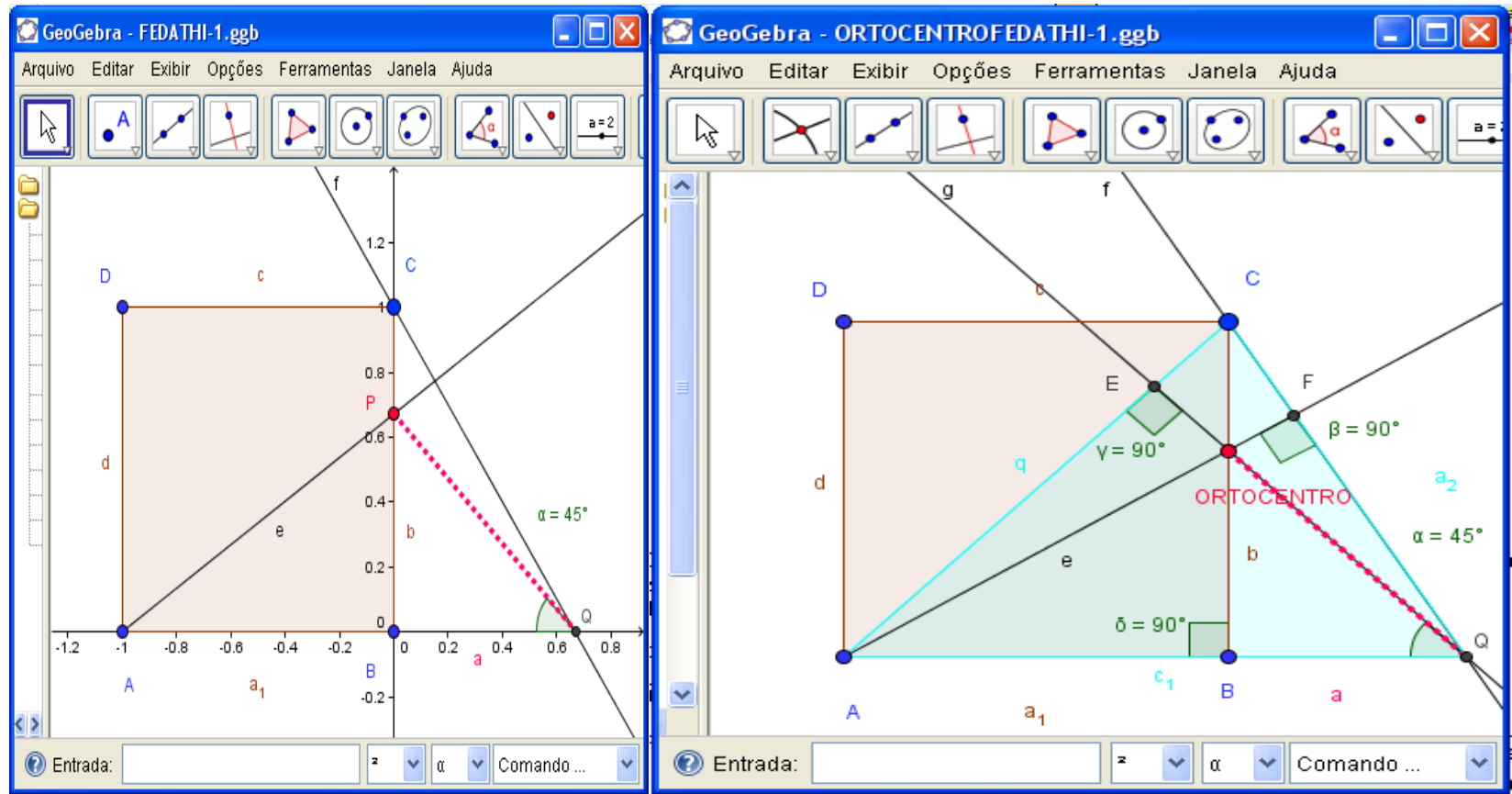

Fonte: Elaboração dos autores.

Vejamos outra situação em que o professor iniciante depara um teste para suas habilidades que envolvem a elaboração de um desenho.

Problema 3. Seja $A A^{\prime}$ uma diagonal de um cubo e sejam $\mathrm{B}, \mathrm{C}$ e $\mathrm{D}$ os vértices do cubo mais próximos de A. Mostre que $A A^{\prime}$ é perpendicular ao plano que contém os pontos $\mathrm{B}, \mathrm{C}$ e $\mathrm{D}$. 
Vamos simular uma situação pouco didática que pode ocorrer. Assim, o professor iniciante pode simplesmente eliminar as etapas previstas na SF e proceder à fase de solução. Argumenta que, tomando o vetor $\overrightarrow{B C}=(-1,-1,0), \overrightarrow{B D}=(-1,0,1), \overrightarrow{A A^{\prime}}=(1,1,1)$. Portanto, fazemos o produto $\left\langle\overrightarrow{A A^{\prime}}, \overrightarrow{B C}\right\rangle=0=\left\langle\overrightarrow{A A^{\prime}}, \overrightarrow{B D}\right\rangle ;$ assim, $\overrightarrow{A A^{\prime}}$ é um vetor ortogonal aos segmentos $B C$ e $\mathrm{BD}$ do plano $B C D$. Segue o resultado. Nesse caso, não houve exploração alguma da percepção e da intuição. 0 raciocínio empregado foi o lógico-matemático.

Embora correto, o aluno pode simplesmente repetir as mesmas cadeias de inferências lógicas descritas há pouco, todavia, sem significado algum. Toda a atividade do estudante reproduz apenas os objetivos preestabelecidos a priori pelo professor e a autonomia do aprendiz não evolui. Assim, para que evitemos uma discussão com pouca perspectiva de continuidade em sala de aula, adaptamos o problema anterior e enunciamos o problema $3^{*}$ que pode ser encontrado em Lima e outros (1999, p. 94).

Problema 3*. Seja $A A^{\prime}$ uma diagonal de um cubo e sejam $\mathrm{B}, \mathrm{C}$ e $\mathrm{D}$ os vértices do cubo mais próximos de $\mathrm{A}$. (a) Mostre que $A A^{\prime}$ é perpendicular ao plano que contém os pontos $\mathrm{B}, \mathrm{C}$ e $\mathrm{D}$; (b) Se $A A^{\prime}$ fura o plano BCD em $\mathrm{P}$, mostre que $\overrightarrow{A P}=\frac{1}{3} \overrightarrow{A A^{\prime}}$.

Fonte: Lima e outros (1999).

O elemento que se distingue agora no problema $3^{*}$ é que, acrescentando-se o item (b), o ponto que se pretende determinar nesta questão será o ponto que representa a intersecção das alturas do triângulo de vértices $B, C$ e $D$, é justamente o ortocentro. Mas isto proporciona ao docente o estabelecimento de uma relação conceitual importante entre o conjunto de problemas abordados. 


\section{CONSIDERAÇõES FINAIS}

Discutimos nesse artigo o ensino de Geometria Analítica. Nesse âmbito de interesse, que possui vínculo importantíssimo com a formação do futuro professor de Matemática, colocamos em evidência os pressupostos da metodologia de ensino chamada Sequência Fedathi. A relevância dos pressupostos filosóficos e epistemológicos desta proposta de mediação didática reside no fato de que o professor iniciante na profissão necessita, além de um bom domínio de conteúdo, de uma visão didático-metodológica daquilo que visa a transmitir, além de uma capacidade avaliativa da própria capacidade de transmissão (transposição) desse saber em sala de aula.

Indicamos também ao longo do texto, o papel importante que a tecnologia pode assumir, todavia, demos a entender que seu uso não deve ocorrer de modo precipitado. A tecnologia pode funcionar como um impulsionador de um raciocínio intuitivo manifesto nas fases iniciais da SF (na resolução dos problemas indicados). Ela não pode condicionar esse ou aquele caminho de modo reducionista. Além disso, a tecnologia, quando mediada de modo adequada, pode ensejar situações de conflito cognitivo, incerteza e, na dependência de sua superação, o aprendiz atinge um patamar superior de aprendizado e entendimento de maior amplitude sobre um assunto particular.

O professor deve, no entanto, se manter vigilante, porquanto a dúvida envolve uma combinação instável de ideias (SAURIAU, 1881, p. 41), e o alcance da estabilidade deve ser consequência de uma atividade autônoma do estudante. Por fim, a partir dos problemas 1 e 2, fornecemos indícios para responder uma questão recorrente no imaginário do professor iniciante: quando devo fornecer as definições matemáticas formais em minha aula? Logo no começo?

Na situação do problema 2, apesar de empregarmos a noção de vetores (que é vista no contexto escolar apenas no Estado do Rio de Janeiro), retardamos a descrição do ortocentro de um triângulo para a última fase da SF. De tal sorte, não precipitamos o momento de definir, como recurso somente na lógica, um objeto ou uma propriedade desconhecida pelos estudantes. O elemento mais importante aqui é o fato de que o professor de Matemática dever ser consciente sobre a natureza de uma definição formal e de que modo sua natureza condiciona/determina sua mediação.

Na fase de prova da SF não se podemos imprimir uma ênfase total no método axiomático. De fato, segundo Brousseau (2002, p. 21), seu uso proporciona um caminho de estudo, tanto para o aluno e seu professor, pois no menor espaço de tempo, se acumula uma grande quantidade de saberes. 
Para se atingir, todavia, uma aprendizagem satisfatória, não podemos seguir um trilho e sim uma trilha.

Por fim, ao evitar a algoritmização no ensino de Geometria Analítica o professor estimula o raciocínio heurístico e intuitivo do estudante, leva-os a compreender que em Matemática pode-se errar várias vezes para se obter um acerto em seguida. Na figura 5, destacamos uma concepção ainda está distante de se tornar oficial e operacionalizada em nos cursos de formação inicial no Estado do Ceará, entretanto, esta figura evidencia o fato de que apenas o domínio de conteúdo não é suficiente para uma boa evolução no ofício de professor. Não obstante, a instrumentação com teorias secundária e terciárias, no que concerne ao fenômeno "ocorrência de uma aula", não produz também efeitos representativos. De outro modo, diante de um quadro deficitário da formação inicial de professores, a preocupação primordial se revela dentro da sala de aula, num momento propriamente dito da mediação de um determinado assunto.

Figura 5. Nossa concepção de domínio de conteúdo e de visão de transmissão do mesmo em sala de aula.

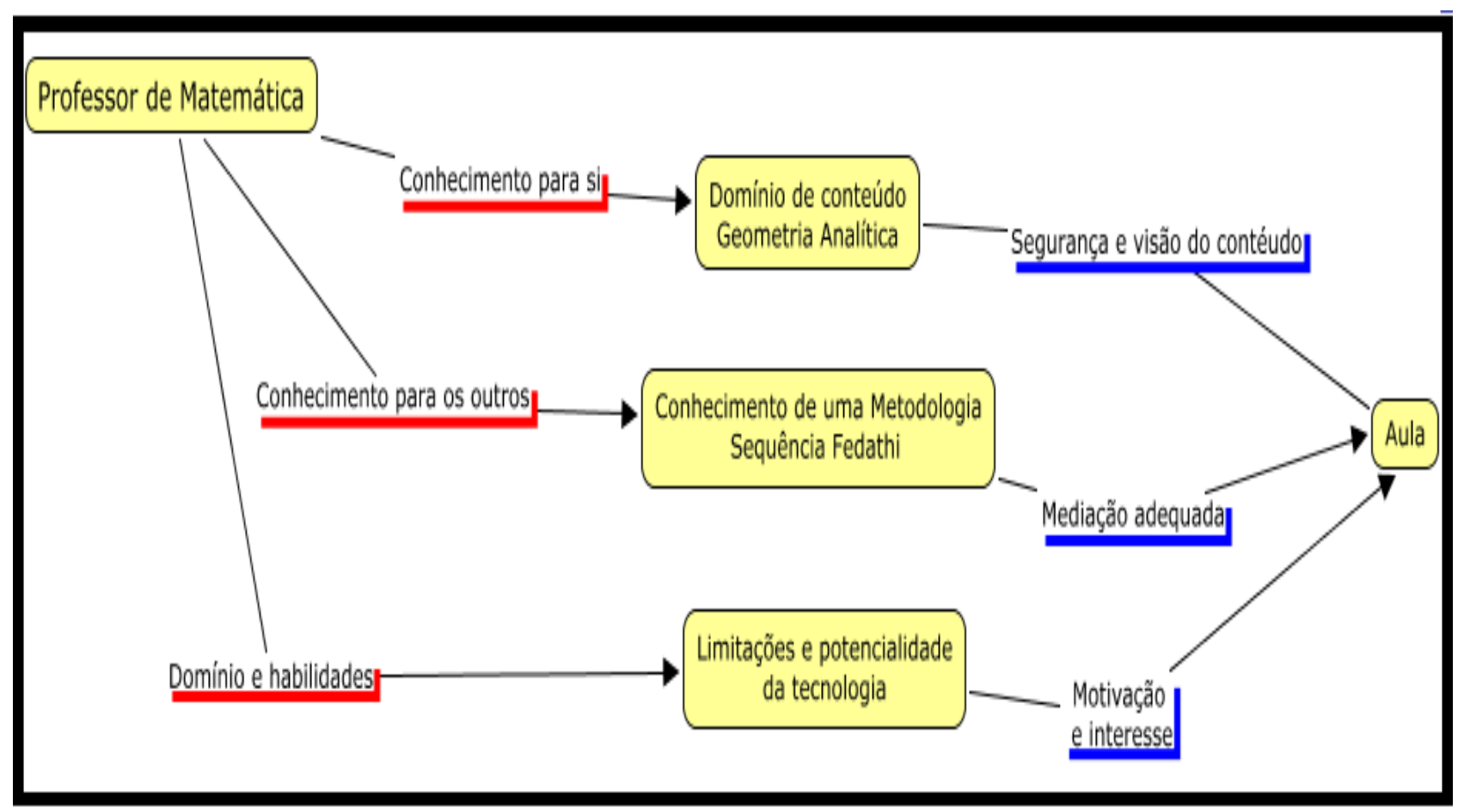

Fonte: Dados da investigação. 


\section{REFERÊNCIAS}

ALVES, Francisco, R. V.; BORGES NETO, Hermínio. A intuição na Sequência Fedathi: uma aplicação no ensino médio. Em: Conexões, Ciência e Tecnologia, vol. 3, n. 1, 30-41, 2009. Disponível em: <revistaconexoes.ifce.edu.br/index.php/ conexoes/issue/archive>. Acesso em: 2 fev. 2014.

; _ـ A existência da Sequência de Fibonacci no campo dos inteiros: uma atividade de investigação apoiada nos pressupostos da Sequência Fedathi. Em: Boletim GEPEM - grupo de estudos e pesquisa em educação matemática, n. 56, 1-6. 2012. Disponível em: <ufrrj.br/SEER/index.php?journal= gepem\&page=index>. Acesso em: 2 jan. 2014.

BARRETO, Marcília Chagas. Uma aplicação da Sequência Fedathi no ensino de Progressões Geométricas e a formação do professor no IFCE. Em: Conexões, Ciência e Tecnologia, vol. 5, n. 1, 9-24, 2011. Disponível em: <revistaconexoes.ifce.edu.br/index.php/conexoes/issue/archive>. Acesso em: 25 dez. 2014.

ANDRADE, Roberto, C. D. Geometria Analítica Plana : praxeologias matemáticas no ensino médio (dissertação de mestrado em educação em Ciências e Matemática). Pará: Universidade Federal do Pará, 2007. Disponível em: <repositorio.ufpa.br/jspui/bitstream/2011/3098/1/Dissertacao_GeometriaAnaliticaPlana.pdf>.

BALACHEFF. Nicolas. Une étude des processus de prevue en mathématiques chez des élèvesde Collège. Thèse de doctorat. Grenoble: Universitá Joseph Fourier, 1988. 602p.

BROUSSEAU, Guy. Theory of Didactical Situations in Mathematics: didactiques de mathemátiques 1970-1990. New York: Klumer Academic Publishers, 2002.

; GIBEL, Patrick. Didactical handling of student's reasoning processes in problem solving situations. Em: Educational Studies in Mathematics, n. 59, 13-58, 2005.

DOS SANTOS, I. N. Explorando conceitos de Geometria Analítica Plana utilizando tecnologias da informação e comunicação. (dissertação de mestrado). Ouro Preto: Universidade Federal de Ouro Preto. 2011. Disponível em: <ppgedmat.ufop.br/arquivos/dissertacoes_2011/Diss_Ivan_Nogueira_dos_Santos.pdf>. Acesso em: 20 maio 2016.

GIBEL, Patrick; BROUSSEAU, Guy. Didactical handling of student's reasoning processes in problem solving situations. Em: LABORDE, Colette; PERRIN-GLORIAN, Marie-Jean; SIERPINSKA, Anna (Edit). Beyond the apparent banality of mathematics classrrom, 13-58, 2005.

GUEDES, Paulo C. Algumas aplicações do software GeoGebra ao ensino da Geometria Analítica (dissertação de mestrado profissional). Vitória do Espírito Santo: UFES, 2013. Disponível em: <bit.profmat-sbm.org.br/xmlui/ bitstream/handle/123456789/587/2011_00376_PAULO_CEZAR_CAMARGO_GUEDES.pdf?sequence=1>. Acesso em: 20 maio 2016

LIMA, Elon Lages. Exame de textos: análise de livros de Matemática para o Ensino Médio. Rio de Janeiro: Sociedade Brasileira de Matemática, 2001.

Geometria Analítica e Algebra Linear. Rio de Janeiro: IMPA, 2005.

e outros. Matemática do Ensino Médio. vol. 3. Rio de Janeiro: Sociedade Brasileira de Matemática, 1999.

POLYA, George. Le découverte des mathématiques: les modèles et méthode generale. Paris: DUNOD, 1967.

. A new aspect of mathematical method. Princeton: Princeton University Press, 1973.

PONTE, João P.; OLIVEIRA, H. Remar contra maré: A construção do conhecimento profissional na formação inicial. Em: Revista da Educação, n. 11, 145-163. 2002. Disponível em: <educ.fc.ul.pt/docentes/jponte/artigos-por-temas.htm>. Acesso em: 18 out. 2014. 
SANTOS, Ricardo S. Tecnologias digitais na sala de aula para a aprendizagem de conceitos de Geometria Analítica: manipulações com o software GRAFEQ (dissertação de mestrado). Porto Alegre: Universidade Federal do Rio Grande do Sul, 2008. Disponível em: <ufrgs.br/espmat/disciplinas/novas_abordagens/modulo_III/pdf/ dissertacao\%20Ricardo.pdf>.

SAURIAU, Paul. Théorie de L’invention. Paris: Félix Alcan, 1981.

SOARES, Susana R. Um estudo histórico do ensino de Geometria Analítica no curso de Matemática da UFJF nas décadas de 1960 a 1970. Juiz de Fora: Universidade Federal de Ouro Preto. 2013. Disponível em: <ufjf.br/mestradoedumat/files/2011/05/Disserta\%C3\%A7ao-final-Susana-Soares.pdf>. Acesso em: 20 maio 2016.

WAGNER, Eduardo. Aplicações de Geometria Analítica. 2006. Disponível em: <video.impa.br/>. Acesso em: 24 dez. 2014.

Aplicações de Geometria Analítica. 2011. Disponível em: <video.impa.br/>. Acesso em: 26 dez. 2014. 